\title{
Application of Wavelet Analysis Model Based on Hilbert Transform in Measles Outbreak Period
}

\author{
Wang $\operatorname{Tan}^{1}$, Qianqian Yang ${ }^{1}$ and Rencong $\mathrm{Nie}^{2, *}$ \\ ${ }^{1}$ School of Mathematical Sciences, Ocean University of China, Qingdao, China \\ ${ }^{2}$ College of engineering, Ocean University of China, Qingdao, China
}

\begin{abstract}
Objective To study the trend of cycle activity of measles epidemic from 1950 to 2014 and establish a model to predict the national incidence of measles in the future. Methods Using the national measles monitoring data from 1950 to 2014, we establish a information database. Then, we set up the wavelet analysis model based on Hilbert transform to study the cycle of measles incidence. Finally, we establish the ARIMA model of measles risk level to predict the incidence of measles by SPSS software. Results Wavelet analysis shows that the outbreak cycle of the incidence of measles is getting longer in the time dimension. ARIMA model analysis shows that national incidence of measles will fluctuate and decline in the next 36 years, which is may related to the improvement of medical standards and people's awareness of the measles prevention. Conclusions The national incidence of measles is declining. It is cyclical and its outbreak cycle is getting longer. Data shows that the incidence of measles will gradually decrease in the future, and gradually achieve the global goal of eliminating measles.
\end{abstract}

\section{Introduction}

Measles is one of the most common acute respiratory infections in children. It is so contagious that it is prone to prevalence in densely populated areas where there is no general vaccine. Therefore, the analysis of the outbreak cycle and the prediction of the incidence of measles have great significance. As far as the health sector is concerned, it helps to formulate optimal prevention strategies and treatment strategies. On the individual side, it helps to better strengthen individual prevention and reduce the probability of disease by injecting vaccines in advance. Significant progress has been made in reducing the incidence and mortality of measles due to the widespread use of the measles vaccine.[1-4]

In recent years, many scholars at home and abroad have conducted extensive research on the incidence and outbreak cycle of measles. There are some common methods such as Fourier analysis, while in this paper we propose a wavelet analysis model based on Hilbert transform. This is a signal time-scale analysis method which has the ability to characterize the local characteristics of the signal in both time and frequency domains. It can perform multi-scale refinement analysis of the signal to obtain the change of each frequency with time and the relationship between different frequencies. Therefore, the outbreak cycle of measles incidence can be accurately analyzed. The commonly used methods for predicting the incidence include time series methods, neural networks, and support vector machines. We propose the ARIMA $(4,1,1)$ model and construct the
ARIMA model through SPSS software to predict the incidence of measles in the next 36 years in this paper. The results prove that the prediction method has high accuracy and real-time performance, and improves the prediction accuracy obviously.

This paper uses the national measles data from 1950 to 2014 to analyze the cycle activity trend of measles by establishing a wavelet analysis model based on Hilbert transform. An ARIMA $(4,1,1)$ model is established to predict the national incidence of measles for the next 36 years. Based on these two results above, relevant departments can obtain the basis for scientific decisionmaking.

\section{Materials and Database}

\subsection{Measles epidemic data}

Data is from the national scientific data sharing platform for population and health. The research object is the national incidence of measles from 1950 to 2014.

\subsection{Construction and analysis of database}

The provincial geographic information database and population measles information database, including regional codes, regional names, years, incidence, deaths, morbidity and mortality $(100,000$ people). Firstly, the wavelet analysis based on Hilbert transform is used to explore the periodic changes of the measles incidence. Secondly, the ARIMA model is constructed with SPSS

\footnotetext{
Corresponding author: tw_math@163.com
} 
software to predict the change of incidence from 2015 to 2050 .

\section{Analysis methods and results}

\subsection{National Measles Epidemic Time Trend}

Figure 1 is a graph of the national incidence of measles over time from 1950 to 2014 . It can be seen intuitively that the case number and incidence of measles nationwide show a downward trend in the time dimension. In addition, the outbreak of measles shows a certain periodicity in the time dimension, and the local extremum also shows a declining trend. This is related to the rapid development of Chinese economy, great improvement of medical conditions and increased awareness of people' $s$ health these years. In order to learn about the outbreak trend of measles in the future, we will use wavelet analysis to analyze the volatility series formed by the national morbidity date.

The case number of measles nationwide shows an upward trend from 1950 to 1957 , but the data is 0 in some provinces, which is suspected to be pathological. This may be due to insufficient statistical capacity at that time. In order to ensure the accuracy of the analysis, we use the data from 1958 and later as the overall study of wavelet analysis.

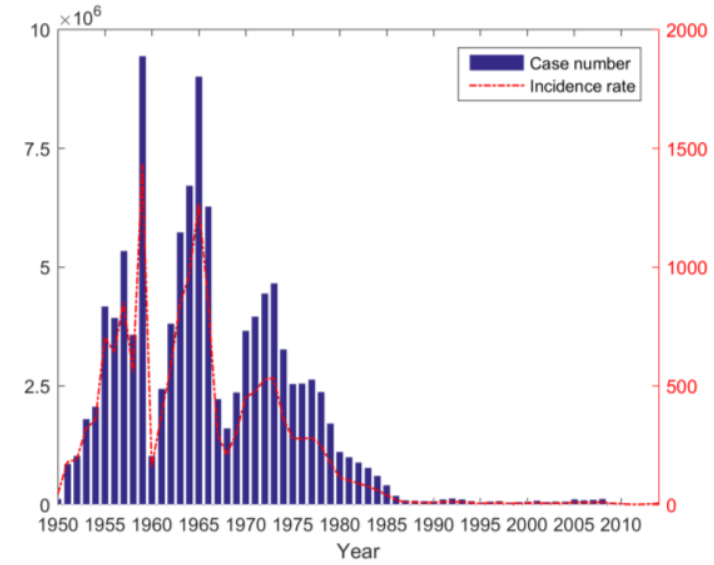

Fig. 1. The case number and incidence of Measles in China.

\subsection{Wavelet analysis model based on Hilbert transform}

The provincial geographic information database and population measles information database, including regional codes, regional names, years, incidence, deaths, morbidity and mortality (100,000 people). Firstly, the wavelet analysis based on Hilbert transform is used to explore the periodic changes of the measles incidence. Secondly, the ARIMA model is constructed with SPSS software to predict the change of incidence from 2015 to 2050 .

\subsubsection{Introduction of Hilbert Transform and Wavelet Analysis}

Hilbert transform is an information transformation used in time signal system to eliminate the effect of amplitude while keeping the frequency and signal transfer cycle unchanged. The Hilbert transform of a continuous time series $f(t)$ is equal to the output response $x h(t)$ of the series after passing through a linear system with impulse response $h(t)=1 / \pi t$. The specific transform formula is as follows :

$$
\begin{gathered}
H[f(t)]=\hat{f}(t)=\frac{1}{\pi} \int_{-\infty}^{+\infty} \frac{f(t)}{t-\tau} d \tau \\
\hat{f}(t)=f(t) * \frac{1}{\pi t} \\
f^{\prime}(t)=\frac{f(t)}{|\hat{f}(t)|}
\end{gathered}
$$

The amplitude of each frequency component in the frequency domain remains unchanged after the Hilbert transform, In order to remove the unnecessary influence of the data fluctuation amplitude on the data cycle analysis, we use Hilbert transform to process the time series of national incidence.

The amplitude of each frequency component in the frequency domain remains unchanged after the Hilbert transform, In order to remove the unnecessary influence of the data fluctuation amplitude on the data cycle analysis, we use Hilbert transform to process the time series of national incidence.

Wavelet analysis is a derivative of Fourier analysis, its basic idea is to express the time series function $f(t)$ with periodic characteristics as a series of successive approximation expressions, all of which are the form after smooth treatment.we can get some useful information such as local cycle by using Wavelet analysis model. In view of this problem background, we use the wavelet analysis model based on the Hilbert transform to analyze the periodic characteristics of national incidence within each year segment.[5]

\subsubsection{Results analysis}

According to the data of the national measles incidence from 1958 to 2014, the data values before and after 1988 quite vary. Therefore, we carry out Hilbert transform around 1988, The results are as follows:

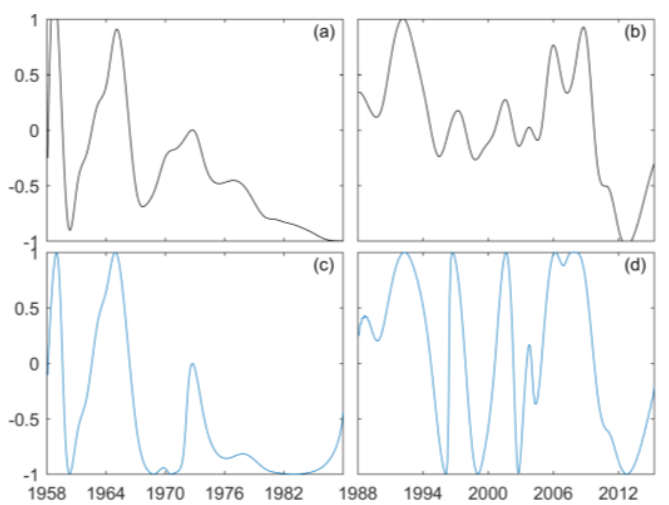

Fig. 2. National incidence sequence Hilbert transform results.

(a), (b) and (c) (d) are respectively the normalized and fluctuated time series before and after 
transformation. According to the Hilbert transform, we can arrive at a conclusion that the periodicity in each year is still apparent, and each period of disease outbreaks varies with years. After fulfilling wavelet analysis of Hilbert transform time series and confidence test, we can gain the following visual rendering.

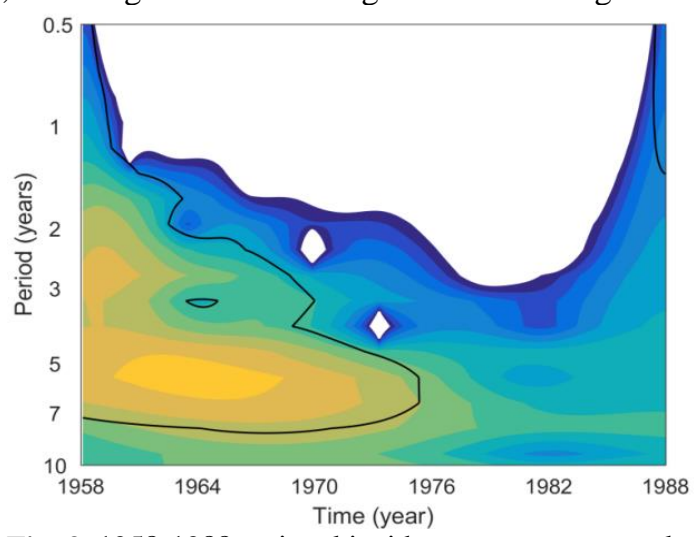

Fig. 3. 1958-1988 national incidence sequence wavelet analysis results.

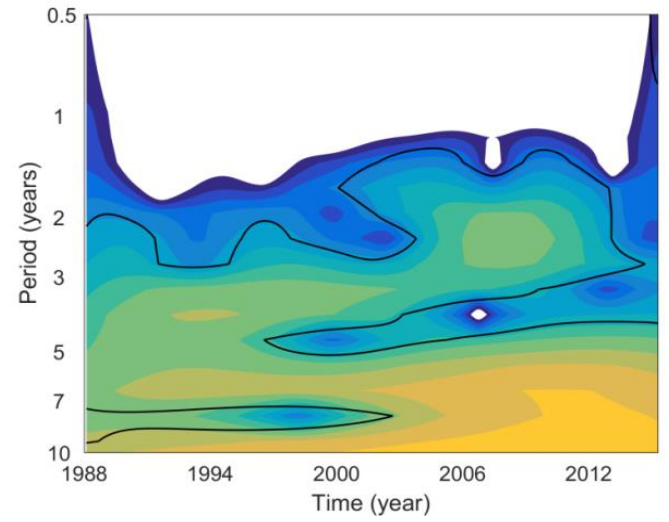

Fig. 4. 1988-2014 National incidence Sequence wavelet analysis results.

Confidence of the place within the black line envelope exceeds $95 \%$. The closer color is to yellow, the stronger signal sequence fluctuations is, the more clear periodic signal is, the more cyclical movement trend performs. On the contrary, the closer color is to the blue, Things are exactly the opposite.Besides, the white is not with confidence at all.

From the above three figures, it can be observed that the periodic signal of 1958-1968 is strong, and the outbreak period of measles is about 5 years at that time; the signal of the period of 1994-2000 is also strong, and measles outbreak period is about 6 years; the signal of the period from 2006 to 2012 is strong as well, and measles outbreak period was about 9 years. It can seen that with the rapid growth of national economic and medical means, the outbreak of the disease is well controlled and the period of outbreak of measles is on the rise of ever-longer-term trends.

\subsection{Wavelet analysis model based on Hilbert transformTime Series Analysis of Measles and National Risk Assessment Based on ARIMA $(4,1,1)$ Model}

\subsubsection{Model Introduction}

Time series is a sequence of ordered data that records the evolution of random events in chronological order. By observing this set of data, looking for the law of data and predicting his future trend are time series analysis. The ARIMA (Autoregressive Integrated Moving Average Model) model is a well-known time series prediction method and it is a method for predicting non-stationary time series.Suppose that the response of the system at time $\mathrm{t}$ is $Y_{t}$, Its value is not only directly related to the response $Y_{t-1}, Y_{t-2}$ of its previous time $t-1, t-2$, but also has a certain correlation with the disturbance $\varepsilon_{t-1}, \varepsilon_{t-2}$ that entered the system at the previous moment. This type of system is called an autoregressive moving average system (ARMA). When the sequence is not stable, the sequence needs to be differentiated first. The model at this time is called the ARIMA model[6].

\subsubsection{Model solving and results analysis}

We use SPSS to construct the ARIMA model. A multiple seasonal ARIMA is established to predict the incidence of measles from 2015 to 2050 , based on the data of national incidence of measles from 1995 to 2014 . The forecast data of 2015-2017 is used as the test data of model accuracy. According to the results, we find that the error between forecast data and actual data in 20152017 is very small, the overall forecast of the incidence of measles in 2018-2050 shows a downward trend. But there is a small area fluctuation in 2025-2029 and the incidence rate increases. Therefore, the relevant departments should attach great importance to the measles in 2025-2029 and take measures. The forecast results are shown in Figure 5 and Table 1.

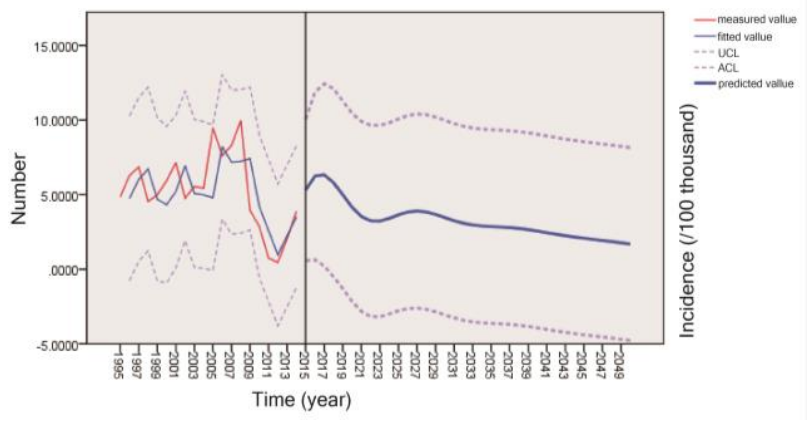

Fig. 5. National incidence forecast results

Table 1. Predicted value of national incidence of measles.

\begin{tabular}{|c|c|c|c|c|c|c|c|}
\hline \multirow[t]{2}{*}{ Year } & \multicolumn{3}{|c|}{ Incidence (/100 thousand $)$} & \multirow{2}{*}{ Year } & \multicolumn{3}{|c|}{$\begin{array}{c}\text { Incidence }(/ 100 \\
\text { thousand })\end{array}$} \\
\hline & $\begin{array}{l}\text { Predicted } \\
\text { value }\end{array}$ & UCL & LCL & & $\begin{array}{l}\text { Predicted } \\
\text { value }\end{array}$ & UCL & LCL \\
\hline 2015 & 5.3105 & 10.0456 & 0.5754 & 2033 & 2.9643 & 9.4605 & 0 \\
\hline
\end{tabular}




\begin{tabular}{cccc|cccc}
2016 & 6.2462 & 11.8572 & 0.6353 & 2034 & 2.8964 & 9.3871 & 0 \\
2017 & 6.3215 & 12.4287 & 0.2143 & 2035 & 2.8592 & 9.3467 & 0 \\
2018 & 5.7999 & 12.1037 & -0.504 & 2036 & 2.8304 & 9.3171 & 0 \\
2019 & 4.9927 & 11.2752 & -1.2898 & 2037 & 2.7915 & 9.2787 & 0 \\
2020 & 4.1512 & 10.4524 & -2.15 & 2038 & 2.732 & 9.2195 & 0 \\
2021 & 3.5382 & 9.9015 & -2.8252 & 2039 & 2.6504 & 9.1372 & 0 \\
2022 & 3.2389 & 9.6528 & -3.175 & 2040 & 2.5523 & 9.037 & 0 \\
2023 & 3.2263 & 9.64 & -3.1873 & 2041 & 2.4465 & 8.9283 & 0 \\
2024 & 3.4069 & 9.8139 & -3.0002 & 2042 & 2.3416 & 8.8202 & 0 \\
2025 & 3.6449 & 10.0691 & -2.7794 & 2043 & 2.2438 & 8.7191 & 0 \\
2026 & 3.83 & 10.2946 & -2.6346 & 2044 & 2.1552 & 8.6276 & 0 \\
2027 & 3.8982 & 10.4 & 0 & 2045 & 2.0747 & 8.5447 & 0 \\
2028 & 3.8358 & 10.3572 & 0 & 2046 & 1.9993 & 8.4671 & 0 \\
2029 & 3.6732 & 10.1968 & 0 & 2047 & 1.9249 & 8.3911 & 0 \\
2030 & 3.4613 & 9.9787 & 0 & 2048 & 1.8487 & 8.3133 & 0 \\
2031 & 3.2516 & 9.7614 & 0 & 2049 & 1.7689 & 8.232 & 0 \\
2032 & 3.081 & 9.5837 & 0 & 2050 & 1.6853 & 8.1468 & 0 \\
\hline
\end{tabular}

\section{Conclusion}

The measles epidemic is an epidemic that the World Health Organization has attached great importance to over the past few years. It is affected by many factors such as the distribution of time and space, population, and economic level. By the wavelet analysis model based on Hilbert, the following conclusions are obtained. The period of disease outbreak in 1958-1968 is about 5 years. The period of disease outbreak in 1994-2000 is about 6 years. The period of disease outbreak in 20062012 is about 9 years. Therefore, with the development of the national economy and medical methods, the outbreak of the disease has been well controlled, and the outbreak cycle of measles is developing to a longer and longer trend..

Due to great importance attached to the measles epidemic and the improvement of modern medical science, the incidence and mortality have been effectively controlled. However, the prevention and control of measles should still be taken seriously by relevant departments. We establish the ARIMA model to predict the incidence of measles in the next 36 years in this paper. According to the results, we find that the prediction of the incidence of measles in 2015-2050 shows a downward trend. But there is a small area fluctuation in 2025-2029, and the incidence rate increases. Therefore, the relevant departments should attach great importance to the measles in 2025-2029 and take measures.

The limitation of this paper is that the current study does not consider the nonlinearity of the measles morbidity sequence. Therefore, the next step should be adding support vector machines and fuzzy predictions, to establish a hybrid model that predicts the linear and nonlinear parts of the morbidity respectively, and enhance the nonlinear prediction ability of the model.
In summary, this paper construct a wavelet analysis model based on Hilbert transform and analyze the periodicity of the outbreak of measles epidemic in the country from 1950 to 2014. It is concluded that the outbreak of measles nationwide is cyclical and the cycle length is getting longer. An ARIMA model is constructed to analyze the national risk of morbidity for the next 36 years. We hope that the results of this study will be seen by more people and make people pay more attention to measles. Relevant departments should attach great importance to reducing morbidity, mortality, and disease aggregation by increasing the measles vaccination rate, developing new measles vaccines, carrying out publicity and education campaigns for measles, strengthening epidemic surveillance and improving medical standards.

\section{References}

1. World Health Organization (2013) Progress in global control and regional elimination of measles, 2000 - 2011. Weekly epidemiological record 88: 29 - 36. Available: http://www.who.int/wer/en. (2013)

2. Aspects G. Global measles and rubella strategic plan, 2012-2020[J]. Genetic Aspects. (2012)

3. Chao $\mathrm{M}$, Lixin $\mathrm{H}$, Jing $\mathbf{M} \mathrm{A}$, et al. Measles Epidemiological Characteristics and Progress of Measles Elimination in China, $2010[\mathrm{~J}][\mathrm{J}]$. Chinese Journal of Vaccines and Immunization, 3: 015.(2011)

4. Ma C, Hao L, Zhang Y, et al. Monitoring progress towards the elimination of measles in China: an analysis of measles surveillance data[J]. Bulletin of the World Health Organization, 92(5): 340347.(2014)

5. Niu Lixiao, Wang Zhengfang, Chuan Chuanzhi, Shang Wenli, Zhang Shengshan. A short-term price mixed forecasting model based on wavelet transform and ARIMA[J]. Research of Computer Applications, 31 (03): 688-691.(in Chinese with English abstract) (2014)

6. Pan Huanhong, Zhu Mengman, Liu Xiaoqing. Application of ARIMA Product Seasonal Model in Forecasting the Incidence of Hand-foot-mouth Disease in Jiangxi Province[J].Modern Preventive Medicine ,45(01):1-4+7.(in Chinese with English abstract) (2018) 